\title{
Programa de actividades físicas para personas mayores de 65 años con Hipertensión Arterial y Cardiopatía Coronaría
}

\author{
Lic. Santiago Ramos Bermúdez \\ Profesor Depto. de Educación Física \\ Universidad Pedagógica Nacional
}

\section{INTRODUCCION}

La actividad física gimnástica y deportiva, como un tratamiento de la Hipertensión Arterial (HTA) y la Cardiopatía Coronaria (CC) viene tomando fuerza debido a numerosos estudios que relacionan la inactividad física con un mayor número de incidentes coronarios y, por otro lado, unos valores bajos de tensión arterial en personas que practican con regularidad las actividades físicas.

El presente trabajo pretende anotar algunas consideraciones y recomendaciones que deben tenerse en cuenta cuando se trabaja con adultos mayores de 65 años, quienes además tengan HTA ó CC.

Debe anotarse que, empero las investigaciones indican la conveniencia de la actividad física, no cualquier ejercicio, ni realizado de cualquier forma, es útil para estos propósitos. No puede afirmarse que "el ejercicio es salud" en todas las circunstancias. De hecho, muchas muertes por infarto y otras lesiones ocurren durante la realización de actividades físicas, en una proporción hasta de 7 a 1 con respecto a los infartos producidos en reposo ${ }^{1}$.

Esto nos obliga a tener ciertos cuidados y a respetarlos principios del entrenamiento, de manera que un elemento tan poderoso como el ejercicio físico no se convierta en un factor adicional de riesgo.

\section{OBJETIVOS}

Plantear a las personas interesadas en el tratamiento no farmacológico de la hipertensión arterial, algunas recomendaciones y consideraciones a tener en cuenta al iniciar un programa de actividades físicas para ese fin.

\section{ASPECTOS MEDICO FUNCIONALES}

El ejercicio físico aumentará la Tensión Arteria (TA), a cifras especialmente elevadas en los pacientes hipertensos y de edad avanzada ${ }^{2}$, por lo que es necesario tomar en cuenta el estado hemodinámico basal del paciente, antes de prescribir el ejercicio como tratamiento. Cuando se hace ejercicio vigoroso, la presión sistólica invariablemente aumenta, mientras que en el trabajo prolongado, el aumento primario de la presión sistólica es seguido por un declive gradual que raramente alcanza el nivel de descanso si e hombre está en buenas condiciones.

En hombres entrenados, estos aumentos no son tan marcados como en los que no han hecho nunca entrenamiento. En un experimento con una carga idéntica el entrenado subió a $150 \mathrm{mmHg}$ de TA, mientras el otro hombre alcanzó $230 \mathrm{mmHg}$, cuando en reposo habían tenido 100 y $105 \mathrm{mmHg}$ respectivamente. 
En trabajos de un consumo de oxígeno de 2.3 lit/ mim., se alcanzaron cifras de 260 $\mathrm{mmHg}$ en personas de pobre condición física. ${ }^{3}$

Aunque el ejercicio eleva de manera brusca la TA estudios cada vez más numerosos indican que el esfuerzo repetido la disminuye, tanto en sujetos normotensos, como en hipertensos. ${ }^{4}$

El ejercicio dinámico repetido disminuye la TA de las siguientes maneras:

1- Reduciendo la noradrenalina plasmática excesiva y el cortisol durante el ejercicio exhaustivo, en los pacientes hipertensos delgados que presentan frecuentemente signos químicos de actividad adrenérgica aumentada. ${ }^{5}$ 6

2- Reduciendo el tono simpático (disminuyendo la frecuencia cardiaca -FC- en reposo y el gasto cardíaco). ${ }^{7}$

3- Posiblemente, produciendo una vaso dilatación crónica y un reajuste en los barorreflejos, reduciendo así la resistencia periférica total. ${ }^{8}$

4- Disminuyendo los niveles de sodio, mediante una mayor sudoración. ${ }^{9}$

5- Relajando la musculatura esquelética. ${ }^{10}$

6- Disminuyendo el peso y la grasa corporal. ${ }^{11}$

Tal ejercicio dinámico debe ser del tipo aeróbico, caracterizado por un volumen (cantidad de kilometraje o duración) elevado, una intensidad (velocidad, frecuencia cardíaca) moderada alrededor del 60 al $80 \%$ de la capacidad aeróbica máxima, a un ritmo estable de trabajo.

Por el contrario, los ejercicios isométricos (elevada contracción muscular en un regimen estático) pueden producir elevaciones considerables de la TA, por lo que se recomienda no realizar esta clase de ejercicios con pacientes hipertensos.

Entonces, como ejercicios adecuados sugerimos: caminar, trotar, nadar, andar en bicicleta, bailar, gimnasia aeróbica y algunos juegos deportivos como el tenis, a condición de que cumplan la intensidad moderada, ritmo estable y duración prolongada.

Estudios de 12 semanas o más, hechos por Bonnano J.A. y Lies, J.E. en 1974, Bayer J.L. y Kash F.W. en 1970, Choquette, G. y Fergusson R.J. en 1973, de Plaen F.J. y Detry F.M. en 1980, Hagberg J.M., Goldberg, A.P. y Eshan A. en 1983,y otros, utilizando las actividades señaladas, mostraron disminuciones significativas en los valores de TA sistólica y diastólica, tanto en reposo como en ejercicio, para normotensos e hipertensos, ${ }^{12}$ con bajas entre 4 y 31 torr. para sistólica y de 8 a 16 para diastólica en reposo, y de 3 a 17 sistólica y 5 a 14 diastólica en ejercicio.

Harter y Goldberg, encontraron una marcada correlación entre el aumento del consumo relativo de oxígeno (V02 max.), luego de un programa de 12 \pm 1 meses, con un grupo de hipertensos en tratamiento de hemodiálisis, consiguiendo aumentos del V02 max. desde $22 \pm 2$ hasta $24 \pm 2$ ml.xkg.xmin-1 $(17 \pm 4 \%)$ así como la duración de la prueba 
(protocolo de Bruce modificado, empezando con $\mathrm{O}^{\circ}$ grados y 1,7 millas por hora, o sea dos niveles abajo de la etapa inicial del protocolo de Bruce, aumentando al grado 5\% y 1.7 millas/hora al cabo de 3 minutos para seguir luego con el protocolo standar de Bruce), desde $743 \pm 50$, hasta $902 \pm 56$ segundos $(26 \pm 8 \%)$, paralelamente a lo cual encontró «una disminución en las presiones sistólica y diastólica, tanto en reposo como durante el ejercicio, en 6 de 9 ejecutantes hipertensos". 13

\section{EL EJERCICIO EN ADULTOS MAYORES DE 65 AÑOS}

El deterioro funcional provocado por la incapacidad física y conservar la independencia y autonomía, son los dos problemas más difíciles en el tratamiento de los mayores de 65 años. $^{4}$

El volumen sistólico en reposo disminuye un $30 \%$ entre los 25 y los 85 años, mientras el gasto cardíaco en reposo baja un $58 \%$ en el mismo lapso. ${ }^{15}$

La velocidad de recuperación de la frecuencia cardiaca, presión arterial, consumo de oxígeno y eliminación de Dióxido de Carbono, después del ejercicio, son mas lentos en los ancianos. ${ }^{16}$

La TA aumenta unos 10 a 40 torr., siendo la mayor parte de este aumento entre los 65 y 70 años. ${ }^{17}$

La captación efectiva de oxígeno es de 2 a 4 mets ( 7 a $14 \mathrm{ml} \times \mathrm{kg}^{1} \times \mathrm{min}^{-1}$ ) en los mayores de 75 años, y de 5 a 7 (17,5 a 24) los de menos de 75 años. Los ancianos "atléticos" que han mantenido una buena condición física alcanzan hasta los 10 mets. ${ }^{18}$ La fuerza muscular disminuye un $20 \%$ al llegar a los 65 años. El ejercicio físico aumenta el contenido de minerales en los huesos y se constituye en factor de protección adicional contra las fracturas, especialmente en mujeres postmenopáusicas. ${ }^{19}$

\section{CANTIDAD Y CALIDAD DEL EJERCICIO REQUERIDO}

Morris y colaboradores, indican que 30 minutos de actividad física al día durante el tiempo libre, a una intensidad de $7,5 \mathrm{k} / \mathrm{cal}$ por minuto o más, puede ser utilizado como sustituto o auxiliar de la actividad física para reducir el riesgo de C.C. ${ }^{20}$

Paffenberger y colaboradores y Magnus y colaboradores, señalan que algunos ejercidos de intensidad moderada ( $70 \%$ más o menos $10 \%$ de la frecuencia cardíaca), como caminar rápidamente, montar en bicicleta o subir escaleras, si son practicados de manera regular durante todo el año, también disminuirán las probabilidades de trastorno coronario.

Para lograr cualquier baja de la frecuencia de CC, es preciso lograr un umbral de 500 k/cal por semana; un gasto energético hasta 2000 kilocalorías semanales proporciona una protección adicional contra el riesgo de CC. Cabe señalar que aumentando aún más todavía esta actividad, no se consigue ninguna protección adicional. ${ }^{21}$ 


\section{ALGUNAS RECOMENDACIONES PARA EL PROGRAMA DE ACTIVIDADES FÍSICAS}

Aceptando entonces que el ejercicio dinámico es una alternativa segura y razonable al tratamiento con fármacos de la HTA, para pacientes no entrenados que padecen de HTA moderada $^{22}$ y que el ejercicio puede atenuar el efecto de otros factores de riesgo de CC, tanto directamente, como a través de la reducción del peso corporal, se puede recomendar:

a. Partir de una evaluación individual del estado médico y tolerancia al ejercido.

b. Seleccionar actividades que desarrollen la resistencia aeróbica: caminar, trotar, nadar, andar en bicicleta, patinar, bailar, aerobics-danza, gimnasia, tenis, baloncesto, fútbol.

c. Dichas actividades deben cumplir con un volumen grande (en relación con la capacidad individual) llegando hasta 30 minutos diarios de ejercicio continuo, con una intensidad moderada (alrededor del $70 \%$ de la capacidad aeróbica y ritmo estable).

d. Controlar la intensidad del ejercido a través de la frecuencia cardiaca. Se puede utilizar el Îndice de Karvonen (1K), así:

$1 \mathrm{~K}=(220$-edad-pulso basal $(\mathrm{PB})) \times 0.6+\mathrm{PB}$ para obtener el límite inferior de la FC durante el entrenamiento aeróbico.

$1 \mathrm{~K}=(220$-edad-PB $) \times 0.8+\mathrm{PB}$ para obtener el límite superior.

Ejemplo: Una persona de 65 años de edad, con un PB (pulso antes de levantarse en la mañana) de 60 pulsaciones por minuto (ppm), tendríamos:

$1 \mathrm{~K}=(220-65-60) \times 0.6+60=117$

ó sea, que 117 pulsaciones por minuto, es el límite inferior.

$1 \mathrm{~K}=(220-65-60) \times 0.8+60=136$

entonces, 136 pulsaciones por minuto es el límite superior.

Significa que durante el entrenamiento aeróbico, esta persona debería trabajar en 117 y 136 pulsaciones por minuto, de manera que si en cualquier momento se toma el pulso (contar las pulsaciones durante 10 segundos y multiplicar por 6), debería tener entre 19 y 22.

Si tiene menos de 19, va muy despacio y deberá apurar un poco el ritmo. Si tiene más de 22, va demasiado rápido y deberá proseguir más despacio.

e. Asegurar buenas condiciones de realización:

- Implementos adecuados: zapatos apropiados, ropas adecuadas, bicicletas, patines, balones, adecuados.

- Instalaciones adecuadas: piso, iluminación.

-Clima :temperatura y humedad tolerables, facilidades para el desplazamiento y acceso a los servicios. 
- Disponibilidad de ayudas médicas en caso de requerirse.

- Motivación y educación permanentes.

- Buena técnica en la ejecución de los movimientos.

f. Asegurar un promedio de 4 sesiones semanales a lo largo de todo el año (y de toda la vida).

g. Ajustar la medicación anti-HTA a las nuevas condiciones de actividad (principalmente diuréticos y betabloqueadores).

h. Incluir ejercicios locales de fortalecimiento muscular para evitar el debilitamiento óseo.

i. Incluir ejercicios de flexibilidad (pasiva) para favorecer la postura y movilidad normales.

j. Respetar las leyes del entrenamiento:

- Individualización del entrenamiento.

- Aumento gradual de acuerdo a la velocidad individual de asimilación.

- Continuidad (crear "hábitos de ejercicio").

- Orientación clara hacia objetivos de salud y no de competencia.

\section{BIBLIOGRAFIA}

1. Thompson P.D., Funk, E.J., Carleton, R.A.. Incidence of jogging in Rhode Island from 1975 trough 1980. J.A.M.A. 247:2535-2538,1982.

2. Amery A., Julius, S., Wiltlock, L.S.: Influence of hypertensión on hemodynamic response to exercise. Circulation. 36:231-237, 1967.

3. Mazorra, Z.R: Actividad física y salud. Editorial científico técnico. Habana. 155-157. 1984.

4. MacMahon, M.I., Palmer, R., Ejercicio e hipertensión. Clínicas médicas de norteamérica. 1.1985: 66-75.

5. de Champlain, J., Coisineau, D., Lapointe, L.,: Symphatetic abnorrnalities in human hipertension. Clin. Exp. Hypertension., 3:417438.1981.

6. Messerli, F., Ventura H., Reisin, C.: Borderline .Hypertension and obesity: two prehypertensive states with elevated cardiac output. Circulation 4 66:55-60 1982.

7. BlomqvistC. G., Saltin B.: Cardiovascular adaptations to physical training. Annu. Rev. Physiol., 45:169-189. 1983. 
8. Sonnerstedt, R., Wasir, H., Hennig, R .: Henníg, R.: Sistematic hemodinamics in mild arterial hipertension before and after physical training. Clin. Sc. Molec. Med.: 45: 145-1499. 1973.

9. Luft, FC and WeinbergerMH..: Sodium intake and essential hypertension. Hypertension: 4 (suppl III) 4-19,1982.

10.Engel B.T., Clasgough M.S., y Gaerder, KR. Behavioral treatment of high blood pressure; follow up results and treatment reccomendations. Psychosom Med. 45: 23-29, 1983.

11.Krotkiewski, M. Mandroukas, K., Sjostrom, L., Effects of long terrn physical training on body fat, metabolism and blood pressure in obesity. Metabolism: 28: 650-658, 1979.

12. Harter, H.R., y Golberg, A.P. Programa de ejercicios para entrenamiento resistencial, modalidad terapéutica eficaz para pacientes en hemodiálisis. Clin. Med. NA: 1: 175-189, 1985.

13.Snok, R. Physical activity and aging. Londres. Croom Helm. 1978.

14.Brandfonbrener, N., Landowne, M. y Schock. N.W. Changos in cardiac output with age. Circulation. 12: 557,566, 1955.

15.Schock, M.M. Physiological aspects of aging in man. Ann. Rev. Physiol.. 4230,287, 1977.

16.De Vries, H.A., and Adams GM: Comparison of exercise responses in old and young men. II Ventilatory mechanics. J. Gerontol, 27: 349-352, 1972.

17.de Vries, H.A. Tips on prescribing exercise regimens for your older patients. Geriatrics. 34: 75-77, 80-81, 1979.

18.Fitzgerald, P. El ejercicio en el anciano. Clin. Med. N.A. 1:205-211. 1985.

19.Hagberg, J.M., Esahi, AA. Beneficial effects of endurance exercise training in adolescent hypertension. Am. J. Cardiol. 45: 489, 1980.

20.Leon, Arthur. Niveles de actividad física y cardiopatía coronaria. Clin. Med. N.A. 1: 5-24. 1984.

21.Paffenberger E., Wing, A. Cronic disease in formes college students. Am ,J. Epidemiology . 108: 161-175. 1981. 
22.McMahon, M, Falber E. op. cit.. 